\title{
Los retos de la formación profesional: la formación profesional dual y la economía del conocimiento
}

\author{
Jesús Martín Rivera \\ Federación de Trabajadores de Enseñanza-Unión General de Trabajadores
} (FETE-UGT)

Resumen: En este artículo se pretende ofrecer una visión heterogénea de la formación profesional, ya que su autor ba sido profesor en centros de educación secundaria y de formación profesional. Actualmente trabaja como técnico adjunto en la Secretaría de Formación Profesional de la UGT de Cataluña, por lo que, además de tener una concepción académica, percibe también la sensibilidad de los agentes sociales. En este artículo, se considera importante la opinión de los sociólogos, en contraposición a aquellos estudios en los que solo se tienen en cuenta los criterios economicistas en lo referente al mundo laboral y la empleabilidad. La sociedad del conocimiento tiene, además, unos retos añadidos, como son la inclusividad y la integración social. La formación profesional y el sistema formativo deben asumirlos; de lo contrario, será un fracaso colectivo. Si se observan datos del Centro Europeo para el Desarrollo de la Formación Profesional (Cedefop, 2014), puede verse que España tiene un número muy bajo de alumnos que cursan formación profesional dual (un 4,3\% si se compara con la media de la Unión Europea, que es del $27 \%$, según datos de 2013). Las proporciones de población adulta en paro y con cualificaciones profesionales muy bajas que participan en programas de formación permanente son mucho más elevadas que las correspondientes al promedio de la Unión Europea.

Palabras clave: formación profesional; formación profesional dual; formación profesional continua; cualificación y competencia profesional; abandono escolar prematuro. 
The challenges of the Vocational Education and Training (VET): Dual VET and the knowledge economy

Abstract: This paper is intended to give a heterogeneous vision of Vocational Education and Training, since the author has been teaching in secondary education and vocational training. He is currently working as a technical assistant at the Secretary of Vocational Education and Training in the UGT of Catalonia, so besides having an academic approach, he also perceives the social agents' sensitiveness. In this paper he emphasizes the opinion of sociologists in contrast to those who only take into account the economic criteria in relation to the labor market and employability. Knowledge society also has some added challenges, such as inclusiveness and social integration. The training system in general, and specifically the Vocational Education and Training should assume it; otherwise, there will be a collective failure. If we take a look to the Cedefop (2014) data, we can see that only a small share of VET students are involved in combined work and school-based training (4,3\% compared with the $E U$ average of 27,0\%). The proportions of older adults, unemployed adults, and adults with relatively low qualifications participating in lifelong learning are all higher than the corresponding EU averages.

Keywords: Vocational Education and Trainin; Dual Vocational Education and Training; Continuous Vocational Education; qualifications; Early School Leaving. 


\section{Introducción, Reto industria 4.0. Por la excelencia}

A finales del siglo xx y principios del siglo xxI, se produjeron una serie de cambios significativos en la sociedad industrial. Existe un gran consenso a la hora de establecer que el conocimiento es y será el motor del mundo globalizado. Actualmente, el concepto de industria 4.0 está adquiriendo una gran relevancia, por lo que uno de los retos en el ámbito de la formación tiene que ser formar, captar y retener el talento que el sistema genera y que, a su vez, necesita para atender a las demandas de las empresas.

En la actualidad, se ha pasado de la producción de bienes y servicios a la producción de servicios de la información. Sin duda alguna, la información es el motor de las principales economías del mundo. Por tanto, una sociedad que quiera ser competitiva tendrá que responder satisfactoriamente a los retos que le plantee la formación de su población, atendiendo a sus necesidades tanto presentes como futuras.

Cabe destacar que la industria no podrá absorber, como antaño, la mano de obra sin cualificar que abandona tempranamente sus estudios, porque cada día requiere profesionales mejor cualificados. Por ello, un país como España, con unos índices tan elevados de abandono escolar prematuro (AEP), tiene que solucionar estos problemas estructurales.

Para cumplir este objetivo deben diseñarse estrategias de capacitación que permitan diversificar la actividad económica a través de la cualificación profesional. La formación profesional (FP) tiene que ser el mecanismo que facilite los cambios indispensables para poder competir con economías más flexibles, como, por ejemplo, las asiáticas (Corea, Japón, China, etc.), o bien otras emergentes que compiten en costes. Esto solo será posible si se promueve y se sabe administrar adecuadamente el conocimiento para ser más competitivos.

Martín de la Fuente va más allá y reclama que para afrontar estos cambios, las empresas deben ofrecerle, además, formación conductual a sus plantillas:

El futuro nos demanda hoy profesionales adecuados para una sociedad del conocimiento, con altísimo valor añadido y con una educación donde lo personal pesa más que lo profesional. La empresa debe aceptar esta situación de introducir cambios culturales a través de programas formativos que preparen a sus líderes para trabajar con conductas y emociones (2014:5-6).

La primera revolución industrial trajo innovaciones mecánicas, como la máquina de vapor o el ferrocarril; la segunda supuso la producción en masa a través de la electrificación; y la tercera popularizó los ordenadores e internet. Por su parte, la industria 4.0 tiene aplicaciones de sistemas ciberfísicos (CPS) e integ- 
ra capacidades de computación, almacenamiento y comunicación. Su principal característica es la interconectividad de los sistemas entre sí, conectados, a su vez, con el mundo virtual de las redes digitales globales y la inteligencia artificial.

Se espera que estos sistemas ciberfísicos (CPS) puedan jugar un papel importante en el diseño y desarrollo de los futuros sistemas de ingeniería desarrollando nuevas capacidades que sobrepasan ampliamente los niveles actuales de autonomía, funcionalidad, facilidad de uso, frabilidad y seguridad cibernética. Los avances en la investigación de CPS pueden acelerarse mediante estrechas colaboraciones entre disciplinas académicas y técnicas de computación, comunicación, control para afrontar los grandes desafíos de las aplicaciones (Baheti y Gill, 2011: 166).

Los CPS pueden aplicarse en múltiples sectores, como la fabricación, la energía, la salud, el transporte, las ciudades inteligentes, etc. Implican cambios vertiginosos que no siempre pueden impartirse en centros formativos convencionales.

Las necesidades específicas de los sectores influidos por los CPS suponen un problema de ocupación para aquellas personas que no están suficientemente cualificadas, por lo que se las excluirá de la posibilidad de ocupar puestos de trabajo altamente especializados que requieran competencia digital y conocimiento de lenguas extranjeras. El Foro Económico Mundial (2016:1) ha presentado un informe nada tranquilizador al respecto, ya que estima que se suprimirán en los próximos años 7,1 millones de empleos y se crearán unos 2,1 millones relacionados con la nueva industria.

Al problema del AEP debe añadírsele el de la baja cualificación de estas personas. Si no se subsanan sus carencias a través de la formación continua, se tendrá a una parte considerable de la población adulta con grandes dificultades de empleabilidad y se le excluirá de la posibilidad de acceder a la industria 4.0. Asimismo, cabe señalar que la industria adolece de un vacío de gente cualificada, lo que ralentiza la economía.

España está lejos de ser uno de los países que más personas tiene realizando formación permanente. 
Gráfico 1. Porcentaje de población de 25 a 64 años que participa en una acción de educación o formación permanente en los países de la Unión Europea

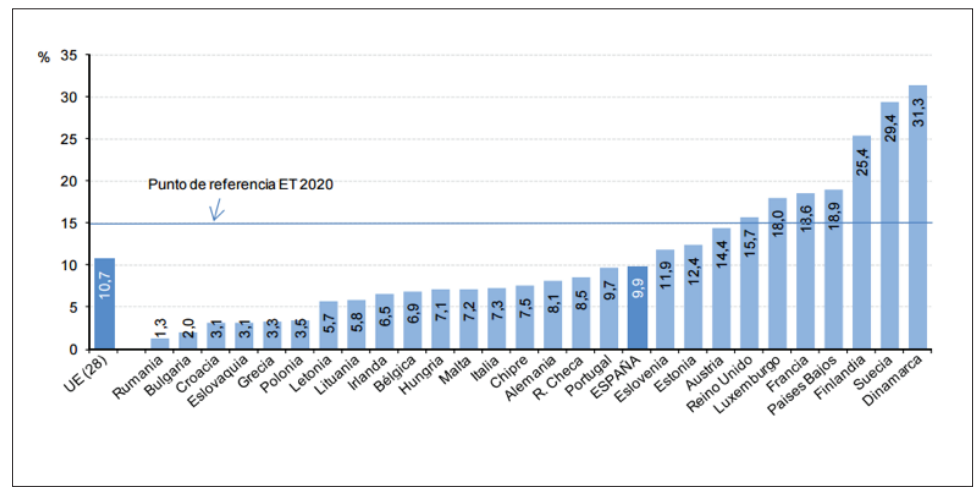

Fuente: Ministerio de Educación, Cultura y Deporte (MECD) (2016).

El reto es cómo incluir a la mayoría de estas personas que abandonaron tempranamente sus estudios y no han seguido ningún tipo de formación en el mercado de trabajo.

¿España está preparada para afrontar el reto de la industria 4.0? ¿Se tiene competencia digital o conocimiento de lenguas extranjeras? ¿El modelo actual de FP responde a estas necesidades? ¿Cuáles son los principales puntos débiles del sistema formativo? ¿Puede solucionarse el AEP?

\section{El papel destacado de la FP}

La FP, además de ser el motor de las políticas de formación, debe ser el eje en el que la población se asegure permanentemente su formación, cualificación, capacitación y, en una sociedad globalizada, internacionalización del conocimiento. Los centros de FP son nodos de transferencia tecnológica entre el mundo de la empresa y los centros educativos. La FP tiene que ser bidireccional, tratar de optimizar los recursos existentes y procurar que la sociedad sea más competitiva $y$, a su vez, más inclusiva.

La FP necesita ser reconocida, promovida en su dimensión real, con toda la transversalidad que esto comporta, ya que este aspecto es crucial para asegurar el bienestar, la prosperidad y la cohesión social frente a los retos presentes y futuros (sociedad del conocimiento, economía 4.0, etc.). 


\section{Conocimiento y reto de aprendizaje}

Entre las capacidades de creación de conocimiento, las más importantes son aquellas que les permiten a los alumnos de hoy en día continuar aprendiendo y actualizándose a lo largo de toda su vida laboral. En este sentido, destaca la capacidad para poder establecer objetivos propios, que las personas sean capaces de evaluar sus puntos débiles y fuertes, de establecer un plan de formación, de identificar los recursos de aprendizaje y de monitorizar su progreso y el de la sociedad en general.

Es preciso, pues, que todas las partes implicadas (familias, orientadores, agentes sociales, etc.) estén bien informadas sobre las oportunidades que ofrece la FP de calidad. Si se tiene una buena y sólida FP inicial, esta servirá para fundamentar el conocimiento de una manera generalista. A través de la formación continua se irá adaptando a las nuevas coyunturas del mercado laboral.

Las acciones formativas deben orientarse a la mejora de las competencias profesionales de la personas en activo, no solamente de los desempleados.

$\mathrm{Al}$ planificar la FP tienen que ofrecerse programas flexibles, que tengan en cuenta las necesidades tanto individuales como de la comunidad y las empresas. Es indispensable que se realice una buena política de comunicación social para que las familias perciban la FP atractiva y práctica, como la vía para la inserción laboral y la capacitación profesional de sus miembros.

La FP no puede diseñarse desde un despacho sin tener en cuenta a los agentes sociales, las empresas, el territorio ni sus necesidades. Debe ser una herramienta que fomente el encaje entre la oferta y la demanda.

\section{La FP dual: la base de la nueva economía del conocimiento}

En los países del centro de Europa, desde hace muchos años, está implantada exitosamente la modalidad dual en la enseñanza profesional, porque sus sistemas formativos tienen una tradición gremial que la favorecen. No puede decirse lo mismo de España, que tan solo tiene un escaso $5 \%$ de estudiantes siguiendo esta modalidad de FP.

La FP dual es la parte más novedosa de la formación profesional en España. Se instauró por medio del Real Decreto 1529/2012, en él se establecen dos modalidades: la alternancia simple y la alternancia dual. Su regulación se ha ido implementando gradualmente en las bases de regulación del Ministerio de Trabajo y Seguridad Social ${ }^{1}$ (ESS/41/2015).

1 Según se recoge en el informe de la Confederación Europea de Sindicatos (CES) (2016: 60-61). 
Esta reforma ha incrementado el porcentaje mínimo de tiempo invertido en formación práctica en el nivel de secundaria superior (FP de grado medio) de un $20 \%$ a un $33 \%$.

Aún es pronto para poder evaluar su implantación, puesto que establecer todas las redes y estructuras de colaboración requiere un tiempo. Sin embargo, sí puede valorarse positivamente, ya que el número de alumnos que siguen esta modalidad se incrementa constantemente año tras año. En el curso 2015-2016, hubo 784540 alumnos en FP, lo que supone un incremento respecto al año anterior de 35952 alumnos (MECD, 2016a) ${ }^{2}$.

\subsection{La FP dual y sus competencias}

A la hora de implantar la FP dual deberían tenerse en cuenta tres factores nada desdeñables: la estigmatización del aprendiz en la estructura productiva, los escasos recursos destinados a la implementación de este sistema educativo y, por último, la sobrecualificación.

La población desempleada tiene una formación profesional o superior que no concuerda con lo que necesita la empresa, por lo que se crea una brecha competencial.

No es necesario aumentar el esfuerzo formativo, sino concentrarlo en las áreas que se prevean con más demanda de mano de obra y en las que se consideren estratégicas por el Gobierno y los agentes sociales.

En los países en los que sí hay políticas de retención de talento y una buena oferta de puestos de trabajo de calidad, las empresas tienen menos dificultades para encontrar personal cualificado. Que las empresas no encuentren los perfiles necesarios para llevar a cabo su función comporta consecuencias graves tanto para el negocio como para el conjunto de la sociedad, lo que redunda en una pérdida de competitividad de la economía.

La FP dual conlleva muchos cambios organizativos respecto al modelo anterior. Supone afrontar nuevos retos: la globalización y la digitalización. Además, cabe destacar otros factores determinantes en su despliegue, como el envejecimiento de la población o la entrada de la universidad en el sistema dual, lo que establece un grado más de competencia. El dinamismo tiene que ser la principal característica del sistema dual en un entorno constantemente cambiante.

Para poder seguir esta dinámica, deben reforzarse las ocho competencias esenciales, especialmente la de lenguas extranjeras y la de las tecnologías de la infor-

2 De los cuales, 16199 alumnos cursan FP dual. Cabe destacar que no se cuenta con cifras homogéneas, puesto que no todas las comunidades tienen en cuenta lo mismo según la información disponible en el siguiente enlace: <www.mecd.gob.es/servicios-al-ciudadano-mecd/dms/mecd/servicios-al-ciudadano-mecd/estadisticas/educacion/indicadores-publicaciones-sintesis/datos-cifras/Datosycifras1516.pdf>. (Consultado el 27/08/2016). 
mación y la comunicación (TIC). Por ello, para seguir resultando atractiva y útil para los jóvenes, la FP dual debe orientarse hacia la internacionalización a través de la globalización y la digitalización.

Con la globalización, la formación debe fundamentarse en las competencias, reforzando especialmente los conocimientos de lenguas extranjeras y las competencias interculturales.

Los resultados y análisis del informe final de la primera encuesta europea de competencias lingüísticas de la Comisión Europea en su sumario ejecutivo (2012: 13) indican que deben mejorarse los sistemas educativos en lo que a adquisición de competencias lingüísticas se refiere: «language competences still need to be significantly improved, and educational systems must step up their efforts to prepare all pupils for further education and the labor market».

Uno de los criterios de contratación para muchas empresas es la experiencia en el extranjero, así como la capacidad para trabajar en equipos plurinacionales.

Los datos publicados por la Comisión Europea de la digitalización de la población adulta muestran que hay grandes desajustes entre los países miembros.

España, según puede observarse en los dos gráficos (gráfico 2), está por debajo de la media europea.

Gráfico 2. Porcentaje de la población de la Unión Europea con competencias digitales bajas o sin competencias y porcentaje de la fuerza de trabajo de la Unión Europea con competencias digitales bajas o sin competencias.

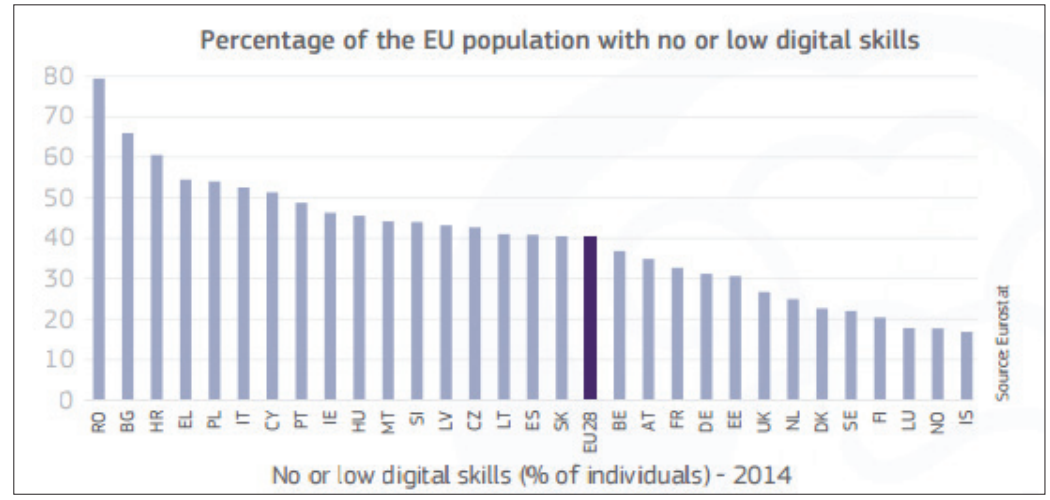




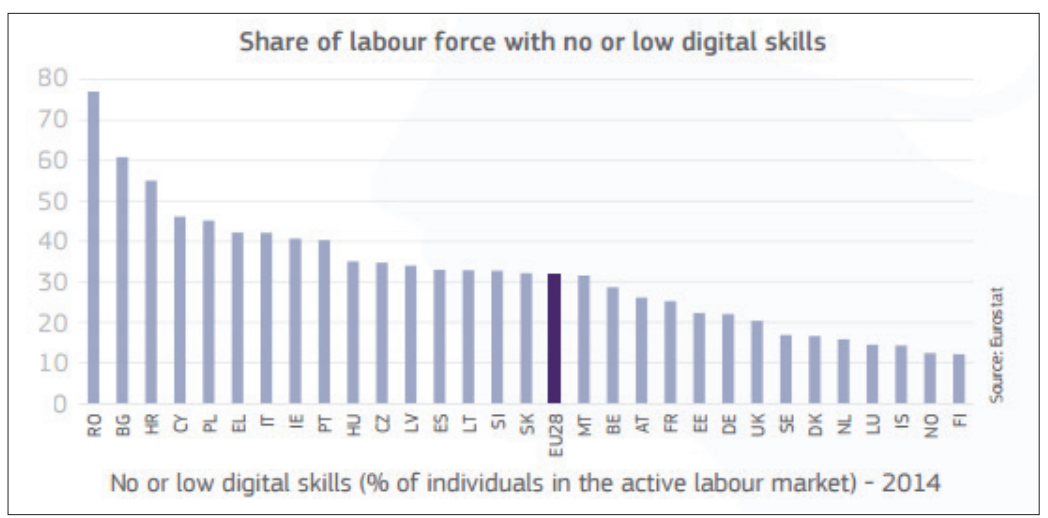

En competencia digital, en 2015, según se desprende de la información aportada en la tabla $1^{3}$, se estudiaron cuatro disciplinas: información, comunicación, resolución de problemas y software.

Tabla 1. Porcentaje de personas con competencias digitales.

\begin{tabular}{|c|c|c|c|c|}
\hline \multicolumn{5}{|c|}{$\begin{array}{l}\text { \% individuals with 'above basic' digital skills, } \\
2015 \\
\end{array}$} \\
\hline & 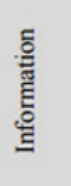 & 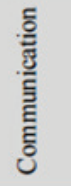 & 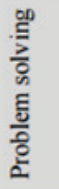 & ᄅ्ञ \\
\hline EU & 65 & 56 & 52 & 39 \\
\hline AT & 71 & 56 & 58 & 48 \\
\hline $\mathrm{BE}$ & 70 & 69 & 57 & 38 \\
\hline BG & 41 & 47 & 24 & 20 \\
\hline $\mathrm{CY}$ & 56 & 57 & 28 & 25 \\
\hline $\mathrm{CZ}$ & 66 & 52 & 48 & 33 \\
\hline $\mathrm{DE}$ & 80 & 64 & 65 & 47 \\
\hline DK & 87 & 71 & 79 & 61 \\
\hline $\mathrm{EE}$ & 79 & 67 & 63 & 46 \\
\hline EL & 57 & 45 & 30 & 28 \\
\hline ES & 64 & 54 & 49 & 43 \\
\hline
\end{tabular}

Fuente: Eurostat.

Si se compara la media de la Unión Europea con España, solo se saca ventaja en software. El reto primordial es evitar que se produzca una brecha digital que propicie que se pierda competitividad, especialmente ante la llamada cuarta revolución industrial.

Según García-Ruíz, Sandoval y De Cos Ahumada, deben conocerse las competencias necesarias:

3 Datos disponibles del año 2015 en la base de datos de la Eurostat, code [isoc_sk_dskl_i] for digital skills. 
[... ] pero principalmente supone el reto de identificar cuáles son las competencias que el ciudadano de esta sociedad tecnológica necesita adquirir para garantizar el desarrollo de todas sus posibilidades, compromisos y actuaciones (2013:5).

\subsection{Gestión de recursos humanos}

La reposición de las plantillas puede concebirse como un problema. Sin embargo, si se planifica adecuadamente es una oportunidad para incrementar su valor añadido, ya que las nuevas generaciones tienen más competencia digital que las anteriores.

A la hora de diseñar modelos formativos debe tenerse en cuenta que no pueden elaborarse planes de formación sin contar con los agentes sociales, ya que estos conocen la realidad de los sectores y las empresas y, por tanto, pueden gestionar mejor estas situaciones.

Las áreas de producción y de comercio evolucionan, se reforman, por lo que se requiere una mayor eficiencia. Hoy en día los ciclos de innovación son más rápidos y los procesos de producción se enfrentan a nuevos retos, como a una mayor flexibilidad y a una rápida capacidad de adaptación. Esto repercute directamente en algunas profesiones, lo que genera, a su vez, nuevos campos de trabajo y nuevos oficios, como informático especializado, gestor de medios, experto en mecatrónica o electrónico de sistemas. Así, las competencias transmitidas en la FP dual deben reformarse y adaptarse a los cambios.

Para planificar y hacer sostenibles los recursos humanos, estos deben gestionarse con flexibilidad.

\subsection{Una FP dual excelente}

La FP dual debe tener una excelencia compartida con la universidad, los cursos en línea masivos y abiertos (CEMA) y los recursos educativos abiertos (REA).

Últimamente, algunas universidades están incorporando a alumnos de FP de grado superior, a quienes les convalidan créditos si hacen carreras de tipo técnico. Greciet, asesora de formación en U-tad, destaca la posibilidad de que los estudiantes de FP puedan acceder a la universidad.

Estamos hablando ya el mismo idioma, de modo que los que hacen FP pueden pasar a la universidad más fácilmente. Aunque los ciclos no son enseñanza universitaria, los alumnos pueden continuar formándose si quieren. Se puede convalidar un mínimo de 30 créditos ECTS - los ciclos de FP se rigen también por este sistemapara acceder a un grado universitario de la misma área de conocimiento ${ }^{4}(\mathrm{xxx}: \mathrm{xx})$.

4 Texto disponible en el siguiente enlace: <www.expansion.com/2013/02/19/entorno/aula_abierta/1361267417.html>. (Consultado el 19/07/2016). 
También se les están convalidando créditos a aquellas personas que por problemas de tiempo o económicos podrían continuar formándose telemáticamente en REA o CEMA. Esta es una modalidad de estudio que ofrece oportunidades de formación y aprendizaje de una manera abierta y gratuita, y que después, algunas universidades convalidan.

El Reino Unido ha intentado crear unos centros de élite para alumnos de entre 14 y 19 años, los llamados university technical colleges, que a diferencia del modelo español pretenden crear una élite competitiva en el ámbito internacional. No obstante, las objeciones a este tipo de actuaciones se han hecho públicas:

Sin embargo, poco se ha hecho para hacer frente a las necesidades de los jóvenes que no están considerados capacitados para acceder a este tipo de educación, y la mayoría ingresa en programas que atraen a estudiantes principalmente de grupos socioeconómicos de clase social baja y en los cuales no se producen cambios significativos (Atkins y Flint, 2015: 36).

Este tipo de iniciativas son acertadas si no son minoritarias. Deben facilitarse en un programa específico dirigido a la mayoría de personas que quieran acceder a una formación, especialmente a las clases sociales más desfavorecidas, que, a su vez, son las que tienen más necesidades. Se les ayudará con becas de movilidad o de formación y con contratos de aprendizaje. En caso contrario, seguirá teniéndose el mismo problema: un estancamiento en la empleabilidad de los jóvenes y los desocupados. Si no se planifica correctamente, se dilatará y acentuará aún más este problema.

\subsection{La agilidad de la FP dual}

Uno de los retos de la FP dual es ser ágil. Un sistema ágil no es frágil. Si los proyectos de formación no son dinámicos, cuando se hayan cumplido los objetivos iniciales, si no se renuevan incorporando cambios, caducarán.

Es preciso que se ejerza un liderazgo. Puede ser compartido por los consejos de dirección o por las direcciones de los centros de formación para prever el agotamiento de los proyectos y, al mismo tiempo, para identificar, en colaboración con los sectores productivos y los agentes sociales, aquellos que puedan iniciarse. Los centros integrados son la solución ideal, aunque no la única.

Para llevar a cabo nuevos proyectos, estos deben responder a las necesidades del territorio. Sin ningún tipo de duda, debe contarse con el apoyo de los agentes sociales y de la Administración educativa, porque sin la flexibilidad adecuada en lo que atañe a la organización de los centros educativos, los proyectos están con- 
denados al fracaso, ya que cada sector tiene unos límites para acoger a alumnos en formación y de inserción laboral.

La FP actual está muy próxima a las empresas y a sus necesidades. Tanto la FP dual como la FP de alternancia simple son los cimientos de una FP de calidad que promueve la excelencia y la empleabilidad de la población.

La FP es fundamental para el futuro y el desarrollo de los países, puesto que forma parte de la estrategia industrial y sectorial. Es indispensable que la formación se planifique junto con estas políticas.

\subsection{La FP dual y las pymes}

La OCDE, en su «Informe de diagnóstico sobre la estrategia de competencias: España 2015», señala lo siguiente:

Un factor que limita la inversión total en I+D en España también puede ser su porcentaje relativamente alto de empresas muy pequeñas, cosa que merma la innovación y la productividad. Las empresas españolas se enfrentan a una serie de obstáculos a la innovación, como la baja tasa de emprendimiento y el acceso restringido al capital riesgo. España podría servirse mejor de los trabajadores altamente cualificados y de las universidades para fomentar la innovación, la productividad y el crecimiento. Esto supondría una reducción de las barreras a la innovación y el emprendimiento y la creación de incentivos para invertir en CBC. También significaría que las empresas españolas tendrían como eje central en sus estrategias de negocio el uso de trabajadores altamente cualificados dedicados a la innovación. Con frecuencia, las microempresas no poseen la capacidad suficiente para formar a estudiantes y colaborar en el desarrollo de sus competencias prácticas. Esto significa que podría resultar difícil para las empresas españolas asimilar grandes cantidades de estudiantes de FP, por lo que los centros de enseñanza de FP podrían tener dificultades para asignar puestos adecuados a sus estudiantes. Si se optimizan y comparten los recursos no habrá salvedad ninguna $(2015 ; 207)$.

Por la misma razón, el nuevo sistema de FP dual manifiesta dificultades para su implantación, ya que España tiene un 90 \% de pymes y cuenta con poca infraestructura inicial. Un estudio de la Universidad de Valencia, coordinado por Maruenda, destaca lo siguiente:

Las principales dificultades del centro formativo para desarrollar FP dual tienen que ver con la localización de las empresas que deseen participar en el programa (50\%) y que cumplan con sus requisitos $(39 \%$ ) y con la falta de recursos para gestionar la burocracia (2015: 19). 


\subsection{La FP dual integrada: mapa de la demanda y la oferta educativa, y los tutores especializados}

La FP dual se presenta desde la Administración educativa como una formación profesional organizada conjuntamente por las empresas y los centros educativos (mediante convenio de colaboración), que actúan de forma coordinada en el proceso formativo de los nuevos profesionales.

La FP dual tiene que estar gestionada por un órgano exclusivo e independiente que garantice una gestión integrada, con capacidad de decisión y formado por el Gobierno, las empresas y los agentes sociales.

La FP dual se centra en mayor medida en la experiencia del aprendizaje: los estudiantes dedican una proporción mayor de su tiempo a trabajar en una empresa, están «empleados», tienen «contrato»y perciben una remuneración por su trabajo.

Entre otros, uno de los objetivos estratégicos de la FP dual es aumentar el encaje entre la demanda y la oferta del mercado laboral a través de la captación de talento, incrementando las vocaciones profesionales e industriales. Para esto, es necesario que la Administración elabore un mapa territorial donde se planifiquen la política y las acciones formativas según las necesidades de cada zona.

Una FP dual de calidad será solo posible si se mejora la orientación profesional y académica en todas las franjas de edad posibles con una tutorización profesionalizada y adecuada tanto en el centro educativo como en el trabajo. Para ello, es preciso que se realicen cursos específicos de formación para estos tutores, así como que exista una coordinación real entre los dos ámbitos: el educativo y el laboral.

\section{Acciones y propuestas}

Un factor determinante para la proliferación de la FP en España es asegurar el compromiso de los agentes sociales para poder ofrecerles a los estudiantes oportunidades de formación práctica. Para conseguirlo, los sectores y los agentes sociales deben estar bien coordinados.

Para afrontar estos retos pueden consensuarse las siguientes diez acciones.

\subsection{Lucha efectiva contra el AEP}

Es curioso que ya en 1981, Schwartz, en su informe al primer ministro francés apuntara a la alternancia, es decir, a la modalidad dual, como una de las soluciones al problema del AEP, puesto que les garantizaría a los jóvenes una cualifica- 
ción profesional y social. Si el poder político y los agentes sociales no toman a tiempo las medidas adecuadas, el problema se acentuará.

La Comisión de la Unión Europea finalmente es consciente de que el problema debe atajarse. Hace falta implementar las reformas necesarias en el sistema tanto educativo como de formación ${ }^{5}$.

Realicen, cuando proceda, reformas del sistema educativo que abarquen todo el espectro de la educación y formación, incluido el aprendizaje no formal, y reconozcan el papel que desempeña el trabajo juvenil, con vistas a reforzar la continuidad estructural, pedagógica, curricular y profesional, facilitar las transiciones, abordar la segregación y las desigualdades en los sistemas educativos y promover medidas que apoyen la evolución y el rendimiento escolar de los alumnos y les motiven para finalizar su educación (Secretaría General del Consejo de la Unión Europea, 2015: 9).

Si se observa la evolución histórica del AEP en la Eurostat ${ }^{6}$, España ha pasado del 40,4 \% en 1992 al 20 \% en 2015, lo que indica de dónde se viene, lo que se ha mejorado y lo que aún queda por mejorar para llegar al ansiado $10 \%$. La oferta formativa debería diseñarse para ser una formación finalista y el mercado la debería concebir como un nivel de cualificación final.

Como se ha visto, España tiene un nivel elevado de población con educación superior, pero globalmente tiene un déficit muy importante de cualificación en los niveles intermedios, especialmente por el escaso desarrollo de la formación profesional, debido a un exceso de población con niveles bajos de educación. A pesar de que la situación ha mejorado últimamente, continúa habiendo un número demasiado elevado de jóvenes con niveles de cualificación bajos, cerca del $20 \%$.

Los estados miembros de la Unión Europea, en la estrategia Europa 2020, se han fijado unos objetivos específicos en los ámbitos formativos y laborales resumibles en los siguientes puntos:

- Conseguir una tasa de ocupación del $75 \%$ de la población de entre 20 y 64 años.

+ Reducir hasta el 10 \% la población de entre 18 y 24 años con una educación secundaria de primera etapa que ni estudian ni se forman - en el caso español, atendiendo al punto de partida, el objetivo es bajar al $15 \%$ -

- Aumentar hasta el $40 \%$ el porcentaje de la generación más joven con estudios superiores completos.

5 Sorprendentemente, el Reino Unido votó en contra de la recomendación para reducir el AEP en 2011.

6 Tabla comparada entre los estados miembros de la Unión Europea sobre el AEP disponible en el siguiente enlace: <ec.europa.eu/eurostat/tgm/table.do?tab=table\&init $=1$ \&language $=$ en $\&$ pcode $=$ tsdsc410\&plugin $=1>$. 
Puede afirmarse que sin niveles de educación postobligatoria generalizados no será posible el funcionamiento de una sociedad basada en el conocimiento, por mucha inversión tecnológica que se lleve a cabo.

A continuación, se reproducirá un resumen de los retos comunicados por la propia Comisión Europea:

Todos los ciudadanos de la UE deben tener la posibilidad de adquirir la amalgama de conocimientos, cualificaciones y capacidades que requieren para obtener un puesto de trabajo independientemente de su edad, género, origen socioeconómico o étnico o de su discapacidad.

Con esta finalidad, los sistemas de educación y formación deben ofrecer la mezcla adecuada de cualificaciones, en particular de competencias informáticas y transversales clave, el dominio de las competencias básicas para analizar los contenidos de los medios de comunicación y la posibilidad de comunicación en una lengua extranjera. También deben garantizar que los jóvenes que han obtenido sus titulaciones de la educación secundaria y terciaria posean las cualificaciones y las competencias necesarias para lograr una transición rápida y adecuada al empleo. La lucha contra el abandono escolar prematuro y las bajas cualificaciones en competencias básicas como la comprensión escrita, nociones elementales de cálculo numérico y de ciencias - especialmente entre los adultos-, constituyen un elemento esencial a efectos de la inclusión, el empleo y el crecimiento. La formación continua debe alcanzar el indicador del $15 \%$ de participación de la población adulta total en el aprendizaje permanente ${ }^{7}$ (2010: 13).

\subsection{Mejora e incremento de la formación permanente}

Con la cifra del $15 \%$ se ha cerrado el punto anterior como cifra aconsejable por la Comisión Europea para 2020. Si la población ha tenido una FP inicial de buena calidad y sólida, entonces sí se optimizará la formación permanente.

Difícilmente puede sostenerse que una persona que solo ha cursado una FP inicial vaya a tener trabajo asegurado para toda su vida. El propósito es que haya el máximo de progresiones en las cualificaciones profesionales escaladas de N1 a N5 en el marco de cualificaciones del Instituto Nacional de Cualificaciones (INCUAL) $\square$ el reto es incrementar la cualificación y que se sea capaz de mejorar el nivel de competencia $\square$. Para conseguirlo, debe potenciarse la concienciación de la necesidad de aprendizaje permanente, ligado a los cambios y las transformaciones constantes en el entorno laboral, especialmente en la sociedad 4.0.

7 Consúltese este enlace: <eur-lex.europa.eu/legal-content/ES/TXT/PDF/?uri=CELEX:52010DC0682\&from $=E N>$. (Consultado el 14/08/2016). 
La cuarta revolución industrial acelera los cambios, por lo que la formación permanente tiene la misma importancia y transcendencia que la inicial. Las habilidades se adquieren con el aprendizaje formal y no formal, por lo que los dos son complementarios e imprescindibles para asegurar la empleabilidad del presente y el futuro.

Fernández Enguita propone una asociación entre la formación inicial y la continua, ya que no se trata de la crisis de la escuela y el retorno al aprendizaje.

[...] porque una paradoja de este proceso es que la necesidad de renovación permanente no hace sino reforzar el carácter crucial de la formación inicial. Más que nunca se torna verdad la vieja máxima: la mejor formación profesional es una buena formación general, siempre que no se tenga la formación inicial por suficiente. El peso creciente de la tecnología en la producción, y en particular de la innovación tecnológica en la competencia implica un desplazamiento progresivo del peso de los componentes de la cualificación, de los más simples a los más complejos (2003:9).

Fernández Enguita (2003: 10) opina que de los tres conocimientos (profesional, científico y operativo), el profesional se impone al operativo y el científico a los otros dos. Por tanto, la carrera profesional comienza con la formación inicial y se prolongará durante toda la vida laboral.

\section{Promoción de la certificación y la cualificación profesional con orientadores}

Esta propuesta está conectada con la anterior. El reto, pues, es desarrollar la capacidad para reciclar y actualizar competencias de un número muy importante de personas, también en aquellos puestos de trabajo con menores expectativas de crecimiento.

La certificación de competencias que los trabajadores han adquirido a través de la experiencia profesional puede ser el revulsivo para completar su formación. Una vez conseguida, pueden optar a los nuevos puestos de trabajo que se generen y, a su vez, satisfacer la demanda de mano de obra cualificada.

Para hacer efectiva esta actuación debe optimizarse la red de orientadores profesionales con la finalidad de asesorar e informar a las personas, tanto ocupadas como no ocupadas, para mejorar su cualificación y capacitación profesional, aprovechando que ahora el sistema ya no es modular, sino que está fijado en unidades de competencia, lo que le da más versatilidad y polivalencia.

Si se lleva a cabo, permitirá una mayor especialización de la mano de obra. Por eso, se deben implementar e impulsar formaciones específicas. Debe aten- 
derse a las necesidades del territorio y adecuarse a los requerimientos del mundo productivo. Es preciso que los centros de FP trabajen con y para su entorno.

[... ] no debe olvidarse que no se puede convertir a los alumnos en mercancía, en productos destinados a uso comercial; es decir, la empleabilidad no debe devenir un bien absoluto a cualquier precio, $y$ hay que pensar en el alumno por lo menos a medio plazo. Los alumnos deberían recibir una formación de valor, no demasiado especializada, de modo que se permita su movilidad entre empresas y que su vida laboral sea lo más variada posible. Para ello, es importante favorecer también una carrera internacional, a través del dominio de los idiomas (Focus Group del IESE, 2014: 62).

España tiene un déficit considerable en la competencia en lenguas extranjeras. Se cuenta con datos de la Unión Europea (28 países en 2011) en los que la media europea presenta mejores estadísticas que España respecto al conocimiento de idiomas extranjeros ${ }^{8}$.

Tabla 2. Porcentaje de personas con competencias en lenguas extranjeras.

\begin{tabular}{l|c|c|c|c}
\cline { 2 - 5 } \multicolumn{1}{c|}{} & \multicolumn{4}{c}{$\%$ individuals with foreign } \\
language competence, 2011
\end{tabular}

Estos datos refuerzan la importancia de que, además de asumir las ocho competencias básicas, deben potenciarse, sobre todo, las lenguas extranjeras por la globalización y la digitalización.

8 Los datos son del año 2011. La fuente corresponde a la base de datos de la Eurostat (code: edat_aes_123). 


\subsection{Mejora de la imagen y la percepción social de la FP para captar a los alumnos que necesita el territorio}

Es una anomalía que en el Eurobarómetro 369 (2011: 10-11), dedicado a la FP, a la pregunta «Have you ever in the past or are you currently taking vocational education and training?», se responda en 15 países que de forma negativa en su mayoría y, en este sentido, destaca Portugal, España (76 \%) y Malta (73\%). La media es del $53 \%$.

Para mejorar el modelo productivo y la cualificación profesional de los españoles debe incrementarse el número de titulados en FP.

Los sistemas de educación deben ser capaces de escolarizar a toda la población joven hasta los estudios postobligatorios y, a su vez, reciclar a la población adulta que no haya conseguido este nivel en el pasado. Los centros integrados con la propuesta de carta de servicios tienen que formarlos, ya que los trabajadores poco cualificados son los más vulnerables en esta sociedad 4.0.

Un dato esperanzador es que, según los datos que figuran en 2014, en lo que a contratación se refiere, 139864 se dedicaban a los contratos para la formación y el aprendizaje y 16199 lo hacían a la formación profesional dual en el sistema educativo, a partir de una base relativamente baja, 106101 y 60584 para el primero, y 9801 y 4292 para el segundo, en comparación con 2013 y 2012, respectivamente. Este aumento se explica por los acontecimientos posteriores a la implementación del nuevo real decreto de $2012^{9}$.

El estatus de la educación no formal debe ser el aumento de su importancia y para conseguirlo plenamente, deben destinarse fondos económicos adicionales. Asimismo, será necesaria una mayor cooperación y colaboración de los agentes interesados en la FP, incluidos aquellos que participan en el sistema nacional de cualificaciones profesionales. Puede fomentarse, por ejemplo, con la formación de agrupaciones de pymes de un sector concreto que necesiten trabajadores con determinadas cualificaciones profesionales.

\subsection{Mejora de la orientación y el asesoramiento (laboral y curricular) en la educación secundaria obligatoria (ESO)}

El objetivo es doble: por un lado, orientar hacia la empleabilidad y polivalencia tanto a jóvenes como a adultos y, por otro, reducir en lo posible el número de estudiantes sobrecualificados. Para ello, se necesitan más orientadores laborales en la ESO, para optimizar el itinerario formativo del alumnado y colaborar con las oficinas del Servicio Público de Empleo Estatal (SEPE) o autonómicas a fin

9 Fuente: CES (2016: 60-61). 
de que informen y orienten adecuadamente a los jóvenes sobre los perfiles requeridos y las diferentes posibilidades que ofrece el mundo laboral.

Se trata de racionalizar la oferta ajustándola a la demanda. Es contraproducente que haya jóvenes que ocupen puestos de trabajo que no requieren el nivel de cualificación que tienen.

En este segmento padecen las consecuencias de lo siguiente:

- Una estructura de pirámide de formación invertida que se desprende de la planificación seguida en el sistema formativo y de la elección de estudios (en torno al $40 \%$ de los jóvenes tienen una titulación de nivel superior).

- En el modelo productivo español no existe, hoy por hoy, un número de puestos de trabajo suficiente que requiera una alta cualificación.

En este contexto, el incremento de los titulados en FP dual permitiría modificar la distribución de la cualificación de la población activa y contribuir a la mejora del modelo productivo.

\section{Disminución de la precarización: un buen país no es un país de bajo coste (low cost)}

España es el único país de Europa que tiene una proporción de población con una titulación mediana inferior. Hay más gente con una titulación superior que con una titulación en enseñanzas intermedias.

A pesar del crecimiento y de la creación de empleo en España, la dificultad para encontrar mano de obra cualificada que se ajuste a la demanda del mercado va en aumento.

\subsection{Necesidad de incrementar la oferta profesionalizadora para demandas 4.0}

En España, debido, en gran parte, a los modelos de formación y cualificación profesional seguidos con anterioridad y a la baja consideración social que han tenido los estudios de FP en la sociedad, hay unas amplias franjas de población activa con una cualificación profesional alta o muy alta y otra franja con una cualificación baja o muy baja que forman la figura de la pirámide invertida, como ya se ha comentado.

A diferencia de otros países, como se ha podido apreciar en los datos de los gráficos de la Comisión Europea facilitados en páginas anteriores, debido a la preferencia que ha habido por los itinerarios educativos más académicos, no se 
dispone de una franja suficiente de población activa con una cualificación profesional intermedia, es más, se está por debajo de la media europea.

En los países con un alto porcentaje de población con cualificación profesional de nivel medio, esta formación se adquiere con los estudios profesionalizadores, cosa que en España aún no sucede. No deja de ser una paradoja que la Comisión Europea aplauda medidas correctoras de anomalías del sistema educativo como la FP básica y algunas fuerzas políticas y sindicales las critiquen porque son «segregadoras». Es incomprensible que la FP básica no permita continuar estudiando directamente en el sistema educativo, como si se hubiese obtenido una graduación en estudios secundarios.

El sistema educativo y de formación debe permitir unas transiciones más flexibles entre los niveles de enseñanza, entre la formación profesional y la educación general, y entre el mercado laboral y las actividades educativas para personas adultas.

Conceptualmente, la FP está diseñada para que los alumnos desarrollen las habilidades prácticas y los conocimientos necesarios para encontrar empleo en una profesión u oficio particular. Para ello, al menos un $25 \%$ del programa debe orientarse hacia una categoría específica de ocupación u oficio. La FP puede estar basada en la escuela, cuando al menos el $75 \%$ de las horas de capacitación se llevan a cabo en el centro de formación y el resto en un ambiente de trabajo, o puede estar basada en el lugar de trabajo, como, por ejemplo, el sistema de aprendices o sistema dual, que se caracteriza porque al menos un $75 \%$ de las horas de capacitación se realizan en la empresa y el resto en la escuela.

\subsection{Necesidad de mejorar la tutorización en los centros de estudio y en los centros de trabajo}

Esto será solo posible si se mejora la orientación profesional y académica en todas las franjas de edad posibles a través de una tutorización profesionalizada y adecuada en el centro tanto educativo como de trabajo. Para ello, es preciso que estos tutores hagan cursos específicos de formación, así como que se coordinen convenientemente los ámbitos educativo y laboral.

Tendrán que garantizarse los recursos humanos y los materiales necesarios, así como la formación, lo que generará una buena red de formadores y fomentará las becas y las estancias para el profesorado.

Cabe señalar que los tutores de empresa son el referente del aprendiz, por lo que tienen que estar formados y preparados específicamente, teniendo en cuenta cada tipología de empresa. Si son pymes, tendrán que mancomunar recursos y gastos creando redes que puedan compartir recursos, experiencia y capacidades. 
Se está realizando un esfuerzo divulgativo al incidir en la idea de que invertir en formación no es un gasto, ya que existe un retorno de la inversión.

El Monitor de la educación y el empleo para España de la Comisión Europea apunta a lo siguiente:

[... la capacidad limitada de absorción de aprendices de las pequeñas y medianas empresas (pymes) y la falta de formación de los mentores en las mismas siguen constituyendo un importante reto para la consolidación de una FP dual de calidad que garantice una mayor empleabilidad (Comisión Europea, 2015: 9).

\subsection{Mejora de la cualificación juvenil}

Los datos facilitados por la Comisión Europea alertan del problema de cualificación profesional de España, que es el segundo país en los niveles tanto más bajo como más alto (terciario).

En 2015, Eslovaquia muestra la incidencia más alta (34,4 \%) de los que tienen cualificación más baja. Es seguido por España (28,9 \%), Grecia y Lituania (26,2 \% cada uno) y Bulgaria (24,7\%). En términos de sobrecualificación, la más alta incidencia se encuentra en Grecia (19\%). Es seguido por España (12,4\%), Chipre (10,7\%), Croacia (8,9\%) y Portugal (8,2\%) (Soldi, 2016:24).

Esto se corrige, según El Consejo de la Unión Europea ${ }^{10}$, si se dota al sistema de flexibilidad para "promover pasarelas flexibles entre la EFP y la educación superior, en el contexto de los marcos nacionales de cualificaciones» (2012:6).

En muchos casos es la consecuencia de no haber finalizado con éxito los estudios obligatorios (entre el $25 \%$ y el $30 \%$ ), o de haber repetido curso en una o más ocasiones (a los 15 años aproximadamente el $40 \%$ ha repetido como mínimo una vez). Esta realidad se traslada al mercado laboral, donde el porcentaje de paro entre los jóvenes es muy superior al de la población que tiene más de 30 años.

El objetivo es incrementar el número de jóvenes que hagan la transición de la escuela al trabajo mediante la FP para frenar el déficit actual de vocaciones industriales y, a su vez, reducir las tasas de desempleo. Este aumento, en ningún caso, debe ser a costa de la calidad del empleo.

Como advertencia, cabe decir que si no está bien renumerada, la FP dual no será atractiva para los jóvenes.

10 Según las «Conclusiones del Consejo, de 26 de noviembre de 2012, sobre la educación y la formación en Europa 2020: la contribución de la educación y la formación a la recuperación económica, al crecimiento y al empleo (2012/C 393/02)». 


\subsection{La FP integrada: creación y fomento de los centros integrados}

Esta última propuesta no es casual: debe ser la síntesis de todo lo expuesto hasta ahora. La FP integrada debe dar respuestas y soluciones a todas las situaciones profesionales, de los 15 a los 65 años. Debe tenerse en cuenta que la FP no solo es la FP inicial, sino también la FP permanente, que se encarga de cualificar y capacitar a personas ocupadas y desocupadas durante su carrera laboral. Este modelo de FP es el que deben ofrecer los centros integrados.

Una buena política formativa tiene que considerar estas dos modalidades de FP como complementarias. La esencia tiene que ser la transversalidad. Por eso, el concepto de centro de formación también muta y hoy en día se están desarrollando los centros integrados de FP, que con su carta de servicios tendrán que abarcar todas las modalidades posibles.

Uno de los retos de la FP es la formación de adultos y la educación, en particular la de aquellos trabajadores con poca cualificación. Debe entenderse como un instrumento que desarrolle y facilite la ocupación de una manera permanente.

Los centros integrados de FP son la solución para suplir las necesidades del sistema, ya que atienden potencialmente a toda la población en edad de trabajar. Para facilitar el aprendizaje permanente, la integración de los sistemas de formación profesional y la incorporación de la población adulta a la formación, los módulos profesionales se organizan en unidades más pequeñas de formación y evaluación llamadas unidades formativas, lo que permite su transversalidad y agilidad.

Socialmente debe visibilizarse que el mejor sistema para mejorar la formación y la cualificación profesional a lo largo de toda la vida laboral es la FP. La modalidad dual tiene que ser el referente capacitador y cualificador por su flexibilidad.

No debe olvidarse que en la FP en general y en la FP dual en particular, la finalidad es asegurar las cualificaciones y las competencias permanentemente.

Si se analizan los datos del informe del Programa Internacional para la Evaluación de Estudiantes (PISA) y las pruebas de la evaluación de competencias de adultos (PIAAC), es decir, se comparan a los jóvenes con la población adulta, se comprueba que hay una estrecha relación de proyección futura: si no se actúa para cambiar esta tendencia, la población que de joven obtuvo malos resultados seguirá sin mejorarlos. Los alumnos que quedaron atrasados en el informe PISA 2000 son los mismos que ocupan los últimos puestos entre la población que ahora tiene entre 28 y 30 años, por lo que se tiene que actuar en algún momento para revertir esa situación. Los centros integrados de FP son ideales para ofrecer las competencias que la población requiere. 
En una FP realmente integrada se da cobertura potencialmente al $80 \%$ de la población en edad de trabajar, entre 18 y 65 años, pero se tiene en cuenta también que la mayoría es población adulta y que su perfil es diferente al de la FP inicial.

\section{Conclusiones}

Como reto principal debe procurarse bajar la tasa de AEP al $10 \%$ en el año 2020 a través de programas eficaces que sirvan, a su vez, para incrementar el número de personas con estudios secundarios postobligatorios y terciarios, y reducir el número de personas con el nivel de estudios más bajo drásticamente.

Si la mayoría de las personas en edad de trabajar adquiere las competencias clave, especialmente en lenguas extranjeras y digitales, podrá formar parte de la industria 4.0 sin exclusión.

La interdisciplinariedad es importante, así como el manejo de las TIC y los medios o las habilidades para trabajar con máquinas y sistemas interconectados.

La FP tiene que ser la red de seguridad contra la precariedad, el revulsivo profesional y la vía alternativa que ofrezca a las personas con diferentes competencias y habilidades una buena opción formativa y laboral.

Se trata, pues, de que los jóvenes aspiren a obtener un nivel de cualificación y estudios más elevado. El sistema integrado les puede ofrecer la oportunidad de progresar y tener opciones de obtener una mejor formación; en caso contrario, en un futuro próximo, tendrán que reciclarse periódicamente en estos centros. Arntz, Gregory y Zierahn (2016:33) alertan de que la cuarta revolución industrial hará que en España se sustituyan un $12 \%$ de los trabajadores por robots, que realizarán los trabajos manuales y repetitivos ${ }^{11}$.

Esto es evitable si se realizan cursos de formación permanente que favorezcan la progresión y empleabilidad durante la carrera laboral.

El modelo ideal de formación debe responder a un modelo productivo de país, sincronizado, basado en estrategias de un plan de actuación, sostenido en el tiempo, que estructure la industria y los sectores que creen ocupación de valor.

Promover centros excelentes para conseguirlo es una buena idea, pero no debe perderse de vista la perspectiva social, inclusiva e integradora de la FP. El sistema fracasará si atiende tan solo a una minoría, por muy especializada que esté. Debe procurarse que la mayoría de la población tenga perspectivas presentes y futuras.

Para acabar con el elevado paro juvenil, con la precariedad y con la falta de competitividad deben ajustarse las demandas del mercado laboral a través de la internacionalización, la adquisición de buenas competencias técnicas y el dominio de idiomas. Se logrará con un modelo educativo y formativo flexible, que actu-

11 El cálculo de los autores (Arntz, Gregory y Zierahn) se basa en las pruebas PIAAC de 2012. 
alice los conocimientos técnicos del profesorado mediante una formación continua y estancias periódicas en empresas.

Tomando como inspiración la iniciativa europea Agenda de nuevas cualificaciones y empleos, mediante una estrategia global (plan industrial y de formación), se apuesta por la transversalidad de las cualificaciones profesionales, la formación permanente y la inclusividad de las personas más desfavorecidas.

Para que la inserción en el mundo laboral sea una realidad, deben promulgarse leyes que hagan posible el objetivo estratégico n. 3 del Consejo Europeo: promover la equidad, la cohesión social y la ciudadanía activa.

Se espera que se cumplan los valores de referencia europeos del anexo 1, que fija unas cifras esperanzadoras ${ }^{12}$.

En España debe considerarse el aprendizaje en el puesto de trabajo como una medida potencialmente eficaz para la inserción profesional de los jóvenes que estén en una gran desventaja social. Existe una falta de experiencia y un know-how específicos en la aplicación de estos programas de aprendizaje exclusivo para la integración profesional de jóvenes vulnerables. Por eso, se aplauden las iniciativas experimentales de integración laboral que se están desarrollando con jóvenes desfavorecidos socialmente en ocupaciones de floristería, soldadura, etc., o las de redes - como, por ejemplo, la Xarxa Innova de la comunidad autónoma de Cataluña- que promulgan la excelencia formativa.

La flexibilidad es la clave para abordar el futuro con éxito. Se necesitarán personas flexibles con facilidad para integrarse en equipos en nuevos entornos, que tengan espíritu emprendedor, que sean activas y capaces de motivar a sus compañeros.

La sociedad y las empresas dinámicas requieren no anclarse ni en el pasado ni en lo aprendido anteriormente.

Una buena formación puede reducir el desequilibrio social; sin embargo, no tenerla, aumenta la desigualdad social.

Se concluye con un dato esperanzador: si se compara España con los países de la Unión Europea, solo aquí ha habido un incremento notable de alumnos que cursan FP, más de un $5 \%$ que el año anterior.

12 Según el anexo 1 de las «Conclusiones del Consejo, de 12 de mayo de 2009, sobre un marco estratégico para la cooperación europea en el ámbito de la educación y la formación ("ET 2020")». 


\section{Bibliografía}

Arntz, M.; Gregory, T.; y Zierahn, U. (2016): «The risk of automation for jobs in OECD countries: a comparative analysis», en OCDE: Social, employment and migration working papers, en línea. $<$ doi: $10.1787 / 1815199 \mathrm{x}>$. (Consultado el 29/08/2016)

Atrins, L. y Flint, K. (2015): «Nothing changes: perceptions of vocational education in England», International Journal of Training Research, 13 (1), pp. 35-48. < doi: dx.doi.org/10.1080/14480220.2015.1051344>.

Baheti, R. y Gill, H. (2011): «Cyber-physical systems», Control systems society, en línea. Disponible en el siguiente enlace: <www.ieeecss.org $>$. (Consultado el 20/08/2016).

Centro Europeo para el Desarrollo de la Formación Profesional (Cedefop) (2014): «On the way to 2020: data for vocational education and training policies», Country statistical overviews update 2013, en línea. Disponible en el siguiente enlace: <bookshop.europa.eu>. (Consultado el 11/08/2016).

Centro para la Investigación en Educación y Formación Continua (CRELL) (2015): Monitor de la educación y la formación de 2015: España, en línea. <ec.europa.eu/education/tools/docs/2015/monitor2015-spain_ es.pdf $>$.

Comisión Europea (2010): Agenda de nuevas cualificaciones y empleos: una contribución europea hacia el pleno empleo, en línea. Disponible en el siguiente enlace: <eur-lex.europa.eu>. (Consultado el 15/08/2016).

- (2012): First European survey on language competences: executive summary, en línea. <crell.jrc.ec.europa.eu/sites/default/files/files/eslc/Executive\%20 summary\%20of\%20the\%20ESLC_210612.pdf >.

- (2013): Reducing early school leaving: key messages and policy support, en línea. <ec.europa.eu/education/policy/strategic-framework/doc/esl-groupreport_en.pdf $>$.

- (2015): Monitor de la educación y el empleo para España, en línea, Luxemburgo: Oficina de Publicaciones de la Unión Europea. < doi: 10.2766/009667>.

- (2016a): «Ten actions to help equip people in Europe with better skills», Press Release Database, de 10 de junio, en línea. <europa.eu/rapid/press-release_ IP-16-2039_en.htm >. (Consultado el 10/07/2016).

- (2016b): Validation of non-formal MOOC-based learning. Disponible en el siguiente enlace: <bookshop.europa.eu>. (Consultado el 15/08/2016).

- (2016c): GROWTH: internal market, industry, entrepeneurship and SMEs, en línea. <ec.europa.eu/growth/sectors>. (Consultado el 30/08/2016). 
Confederación Europea de Sindicatos (CES) (2016): Marco europeo de calidad para el aprendizaje: propuesta de los sindicatos europeos, en línea, ETUC. $<$ www.etuc.org $>$.

Consejo de la Unión Europea (2009): «Conclusiones del Consejo, de 12 de mayo de 2009, sobre un marco estratégico para la cooperación europea en el ámbito de la educación y la formación ("ET 2020") (2009/C 119/02)», Diario Oficial de la Unión Europea, de 28 de mayo, en línea. Disponible en el siguiente enlace: <eur-lex.europa.eu>.

- (2011): «Conclusiones del Consejo sobre el papel de la educación y de la formación en la aplicación de la estrategia Europa 2020 (2011/C 70/01)», Diario Oficial de la Unión Europea, de 4 de marzo, en línea. Disponible en el siguiente enlace: <eur-lex.europa.eu>.

- (2012a): «Conclusiones del Consejo, de 26 de noviembre de 2012, sobre la educación y la formación en Europa 2020: la contribución de la educación y la formación a la recuperación económica, al crecimiento y al empleo (2012/C 393/02)», Diario Oficial de la Unión Europea, de 19 de diciembre, en línea. Disponible en el siguiente enlace: <eur-lex.europa.eu>.

- (2012b): «Informe conjunto de 2012 del Consejo y de la Comisión sobre la aplicación del marco estratégico para la cooperación europea en el ámbito de la educación y la formación (ET 2020): educación y formación en una Europa inteligente, sostenible e inclusiva», Diario Oficial de la Unión Europea, de 8 de marzo, en línea. Disponible en el siguiente enlace: <eur-lex.europa.eu>.

- (2016): Strategic framework - Education E training 2020, en línea. Disponible en el siguiente enlace: <ec.europa.eu>. (Consultado el 12/08/2016).

Consejo Europeo (2009): Conclusiones del Consejo, de 12 de mayo de 2009, sobre un marco estratégico para la cooperación europea en el ámbito de la educación y la formación («ET 2020»). Disponible en el siguiente enlaceः <eur-lex.europa. $\mathrm{eu}>$.

- (2011): Council conclusions on the role of education and training in the implementation of the «Europe 2020» strategy. Disponible en el siguiente enlace: <eur-lex.europa.eu>.

Eurostat (2015a): «Number of foreign languages known (self-reported) by educational attainment level», Base de datos, en línea. <ec.europa.eu/eurostat, code: edat_aes_123>.(Consultado el 11/08/2016).

- (2015b): «Number of foreign languages known (self-reported) by educational attainment level», Base de datos, en línea. <ec.europa.eu/eurostat, code: isoc_sk_dskl_i $>$.(Consultado el 11/08/2016). 
Eurostat (2016): «Early leavers from education and training», Base de datos, en línea. <ec.europa.eu/eurostat, code:tsdsc410>.

Fernández Enguita, M. (2003): «Prólogo», en I. Brunet y Á. Belzunegui* Flexibidad y formación. Una crítica sociológica al discurso de las competencias, Barcelona: Icaria.

Foro Económico Mundial (2016): «The future of jobs employment: skills and workforce strategy for the fourth industrial revolution», Executive summary: the future of jobs and skills, en línea. <www3.weforum.org/docs/ WEF_FOJ_Executive_Summary_Jobs.pdf $>$.

Fundación Tekniker (2016): Sistemas ciber-físicos, en línea. <www.tekniker. es/es/sistemas-ciber-fisicos $>$ + (Consultado el 10/08/2016).

García-Ruíz, R; Sandoval, Y*; y De Cos Ahumada, C. (2013)ः «La educación mediática en la formación profesional. Propuesta de inclusión», Edmetic: Revista de Educación Mediática y TIC, 2 (2), pp. 37-55.

Gil, A. (2013): «La universidad apuesta por la formación profesional», Diario Expansión, en línea. <www.expansion.com/2013/02/19/entorno/aula_ abierta/1361267417.html>. (Consultado el 19/07/2016).

Martín de la Fuente, J. y García Lombardía, P. (coords.) (2014): Armonizar la educación con el empleo en España: un reto a 5 años, en línea. Disponible en el siguiente enlace: <www.iese.edu $>$.

Ministerio de Educación, Cultura y Deporte (MECD) (2016a): Datos y cifras curso escolar 2015-2016, en línea, Madrid: Secretaría General Técnica Subdirección General de Estadística y Estudios, MECD. Disponible en la siguiente referencia: NIPO línea 030-15-167-2. (Consultado el 27/08/2016).

- (2016b): Sistema estatal de indicadores de la educación 2016, en línea. Disponible en el siguiente enlace: <www.mecd.gob.es/dms/mecd/servicios-al-ciudadano-mecd/estadisticas/educacion/indicadores-publicaciones-sintesis/ sistema-estatal-indicadores/SEIE_2016.pdf>.

Organización para la Cooperación y el Desarrollo Económicos (OCDE) (2015): «Informe de diagnóstico de la estrategia de competencias: España 2015», Skills strategy, informe de diagnóstico: España 2015, en línea. $<$ www.oecd.org/skills/nationalskillsstrategies/Spain_Diagnostic_Report_ Espagnol.pdf $>$. (Consultado el 28/08/2016).

Pin, J. R.; Roig, M.; Susaeta, L.; y Apascaritei, P. (2015): La formación profesional dual como reto nacional, Pamplona: IESE Business School y Universidad de Navarra.

Roca Сово, E. (2010)ः «El abandono temprano de la educación y la formación en España», Revista de Educación, 1, pp. 31-62. 
Saniter, A.; Tūtlys, V*; Volpato, K*; Šiožinienė, K*; Marhuenda, F; Thiel, G.; Mahood, E.; y Toscano, I. (2015): Set of experimental modules of vocational integration developed on the basis of the identified competences needed for handling the work processes. Disponible en el siguiente enlace: <www. sodapprent.eu>.

Schwartz, B. (1981): Linsertion professionnelle et sociale des jeunes, París: La Documentation Française.

Secretaría General del Consejo de la Unión Europea (2015)ः «Conclusiones del Consejo sobre reducción del abandono escolar prematuro y el fomento del rendimiento educativo», Conclusiones del Consejo, de 23 de noviembre de 2015, en línea. Disponible en el siguiente enlace: <data.consilium. europa.eu/doc/document/ST-14441-2015-INIT/es/pdf>. (Consultado el 01/08/2016).

Soldi, R.; Cavallini, S.; Friedl, J; Volpe, M.; y Zuccaro, C. P. (2016): A new skills agenda for Europe, European Union, en línea. <cor.europa.eu/ en/documentation/studies/Documents/New-Skills-Agenda-Europe.pdf $>$. (Consultado el 14/08/2016). 